\title{
Clinical Isolates of Candida guilliermondii Include Candida fermentati
}

\author{
ROSARIO M. SAN MILLÁN, ${ }^{1,2}$ LUNG-CHI WU, ${ }^{1}$ IRA F. SALKIN, ${ }^{3}$ AND PAUL F. LEHMANN ${ }^{1 *}$ \\ Department of Microbiology and Immunology, Medical College of Ohio, Toledo, Ohio 43699-0008'; \\ Departamento de Microbiologia, Facultad de Medicina, Universidad del País Vasco-Euskal Herriko Unibertsitatea, \\ Bilbao, Spain ${ }^{2}$; and Wadsworth Center for Laboratories and Research, New York State \\ Department of Health, Albany, New York 12201-0509 ${ }^{3}$
}

\begin{abstract}
Clinical isolates of Candida guilliermondii that were investigated by isoenzyme and randomly amplified polymorphic DNA analyses represented two distinct species. The two species were distinguished on the basis of delayed fermentation of galactose. The larger group of isolates was closely related to the anamorph $C$. guilliermondii ATCC $6260^{\mathrm{T}}$ (T = type strain) and its teleomorph, Yamadazyma (= Pichia) guilliermondii ATCC $4^{46036}{ }^{\mathrm{T}}$. The remaining group, whose members fermented galactose, was very similar to Candida fermentati CBS 2022, which had for many years been placed in synonymy with $C$. guilliermondii. Three additional groups were represented by individual strains; these strains included $C$. guilliermondii var. soya ATCC 20216, which was found to represent Yamadazyma ohmeri. The type strain of $Y$. guilliermondii is redefined.
\end{abstract}

Candida guilliermondii (Castellani) Langeron et Guerra was described by Castellani as a yeast that was obtained in culture from expectorated material associated with tea factory cough (bronchomycosis) in Sri Lanka (8). It was first named Endomyces guilliermondii and was discriminated from other yeasts on the basis of a physiological profile which included failure to ferment fructose, maltose, and galactose. Endomyces negrii, which was later brought into synonymy (18), was also obtained from individuals with bronchomycosis but differed from $E$. guilliermondii by weakly fermenting galactose $(8,9)$. Mating of isolates showed that the teleomorph of $C$. guilliermondii was an ascomycetous yeast, Yamadazyma (= Pichia) guilliermondii (41). This relationship was confirmed by the demonstration of DNA complementarity between the type strains representing the anamorph and the teleomorph (17).

Since $C$. guilliermondii was first described, a considerable number of yeasts with somewhat similar phenotypes have been reported and, in many cases, have been assigned new names. These yeasts include isolates obtained from clinical and environmental sources and isolates that are being investigated for the production of riboflavin or citric acid (16). Taxa which have been brought into synonymy with $C$. guilliermondii have been listed previously (2), and over the years the taxonomic relationships of these taxa to $C$. guilliermondii have been summarized in a variety of monographs $(13,25,26,39)$. In some cases, cultures of the type or authentic strains no longer exist; taxa which fall into this category include $E$. negrii (see above), Monilia pseudoguilliermondi (= Monilia guilliermondi var. pseudoguilliermondi) (which differed in its ability to clot milk and was not accepted as a distinct species by Diddens and Lodder [13]), Candida paranensis, Monilia muhira, and Monilia lustigi. Other taxa that have been brought into synonymy are represented by living cultures.

Recently, Bai studied a limited selection of isolates identified as $C$. guilliermondii and was able to identify two groups of organisms which differed in their electrophoretic karyotypes and DNA base compositions (1). Bai used the differences in these characteristics to define Candida fermentati (Saito) Bai as a species distinct from C. guilliermondii. However, he was not

\footnotetext{
* Corresponding author. Mailing address: Department of Microbiology and Immunology, Medical College of Ohio, P.O. Box 10008, Toledo, OH 43699-0008. Phone: (419) 381-5424. Fax: (419) 381-3002. E-mail: Lehmann@opus.mco.edu.
}

able to detect phenotypic characteristics that were useful for distinguishing the two species, and he did not examine all of the taxa that had been brought into synonymy with $C$. guilliermondii.

In this study, we used isoenzyme and randomly amplified polymorphic DNA (RAPD) profiles to analyze a panel of clinical and environmental isolates that have been named $C$. guilliermondii. We found that there is substantial genetic heterogeneity within this set of organisms and that $C$. fermentati is not unusual in clinical specimens. The synonyms of $C$. guilliermondii and $C$. fermentati are defined below, and a phenotype that is based on fermentation of D-galactose appears to be useful for distinguishing these species. In addition, we describe additional groups within the panel of strains identified as $C$. guilliermondii.

(Some of the results of this study have been presented previously [34].)

\section{MATERIALS AND METHODS}

Fungi. Isolates that were originally identified as $C$. guilliermondii are listed in Table 1. These organisms were obtained from the American Type Culture Collection (ATCC), the Centraalbureau voor Schimmelcultures, the Agricultura Research Service Culture Collection at the National Center for Agricultura Utilization Research, U.S. Department of Agriculture, Peoria, Ill, the collection at the New York State Department of Health, Albany, and Donald G. Ahearn, Georgia State University, Atlanta. The basic methods used to confirm the identities of the strains were analysis of morphology following growth on Sabouraud and cornmeal agar media and growth in a API 20C kit (bioMérieux Vitek Hazelwood, Mo.) to obtain a carbohydrate assimilation profile (7).

Only isoenzyme profile analyses, API 20C profile analyses, and fermentation tests with glucose and galactose were performed for strains MCO 1557 through MCO $1563^{\mathrm{T}}(\mathrm{T}=$ type strain). The remaining strains were investigated for these characteristics and for additional properties by using the methods described below.

Isoenzyme analysis. An isoenzyme analysis in which native discontinuous polyacrylamide gel electrophoresis was used was performed by previously described procedures $(15,19,20)$. Briefly, isolates were inoculated into $250-\mathrm{m}$ Erlenmeyer flasks containing $100 \mathrm{ml}$ of broth (yeast nitrogen base without amino acids [YNB] [Difco Laboratories, Detroit, Mich.] supplemented with $0.1 \mathrm{M}$ sucrose and $0.03 \mathrm{M}$ mannitol) and incubated in a rotatory shaker for 3 days a $28^{\circ} \mathrm{C}$ and $150 \mathrm{rpm}$. Washed yeast cells were then broken with glass beads in the presence of $2.0 \mathrm{mM}$ phenylmethylsulfonyl fluoride and centrifuged at $13,000 \times$ $g$. The supernatants were used as the sources of enzymes, and their protein contents were assayed by the method of Bradford (5)

The chromogenic reagents used to detect enzyme activity were those listed by Lin et al. (24). The enzyme activities analyzed by using $7.5 \%$ (wt/vol) polyacrylamide gels were malate dehydrogenase (EC 1.11.37) (amount of protein loaded $350 \mu \mathrm{g} / \mathrm{gel}$ lane), glucose-6-phosphate dehydrogenase (EC 1.1.1.49) $(200 \mu \mathrm{g} / \mathrm{ge}$ lane), $\alpha$-glucosidase (EC 3.2.1.20) (200 $\mu \mathrm{g} /$ gel lane), $\beta$-glucosidase (EC 3.2.1.21) (350 $\mu \mathrm{g} / \mathrm{gel}$ lane), alkaline phosphatase (EC 3.1.3.1) (350 $\mu \mathrm{g} / \mathrm{gel}$ lane), acid phosphatase (EC 3.1.3.2) (350 $\mu \mathrm{g} / \mathrm{gel}$ lane), and $\alpha$-esterase (EC 3.1.1.1) (350 
TABLE 1. Strains of $C$. guilliermondii used in this study

\begin{tabular}{|c|c|c|c|c|}
\hline Strain & Other designation ${ }^{a}$ & Taxon & Origin & API $20 \mathrm{C}$ profile \\
\hline \multicolumn{5}{|l|}{ Group I strains } \\
\hline $\mathrm{MCO} 1442^{\mathrm{T}}$ & $\operatorname{ATCC~} 6260^{\mathrm{T}}$ & C. guilliermondii & Expectoration from bronchomycosis & 6772373 \\
\hline MCO 1459 & ATCC 9058 & & Acidophilous milk & 6776373 \\
\hline MCO 1460 & ATCC 20283 & C. parapsilosis var. tokyoensis & $?$ & 6776373 \\
\hline MCO 1461 & ATCC $22017^{\mathrm{T}}$ & C. guilliermondii var. japonica & Soil, Japan & 6776373 \\
\hline MCO 1462 & ATCC $26547^{\mathrm{T}}$ & C. parapsilosis var. tuxtlensis & Sugar- and grain-based beverage, Mexico & 6776371 \\
\hline MCO 1463 & ATCC 56822 & & Human urethra, Poland & 6772273 \\
\hline MCO 1467 & ATCC $42715^{\mathrm{T}}$ & C. mamillae & Nipple of nursing bottle, Japan & 6776373 \\
\hline MCO 1525 & ATCC 58070 & Y. guilliermondii & ? & 6776373 \\
\hline MCO $1536^{\mathbf{T}}$ & ATCC $46036^{\mathrm{T} b}$ & Y. guilliermondii & Ulmus americana frass & 6776373 \\
\hline MCO 1558 & NRRL Y-2083 ${ }^{\mathrm{Tc}}$ & Blastodendrion artzi & Blood & 6776373 \\
\hline MCO 1562 & CBS $2024^{\mathrm{Tc}}$ & Blastodendrion krausi & Ulcer of horse & 6776373 \\
\hline MCO 1560 & NRRL Y-17140 $0^{T C}$ & C. amidevorans & Soil, Italy & 6776373 \\
\hline MCO 1557 & NRRL Y-2081 ${ }^{\mathrm{T} c}$ & $\begin{array}{l}\text { Candida melibiosi var. melibiosi } \\
\quad(=\text { Mikroanthomyces alpinus })\end{array}$ & Gentiana imbricata flower & 6776373 \\
\hline MCO 1559 & NRRL Y-12774 ${ }^{c}$ & Torulopsis xylinus & Mulberry bush & 6776373 \\
\hline MCO 1539 & NYDOH $267-83$ & & Clinical & 6776373 \\
\hline MCO 1540 & NYDOH 374-84 & & Clinical & 6776373 \\
\hline MCO 1541 & NYDOH $607-84$ & & Clinical & 6776373 \\
\hline MCO 1542 & NYDOH $244-85$ & & Clinical (leg ulcer) & 6776373 \\
\hline MCO 1543 & NYDOH 693-85 & & Clinical (ear) & 6776373 \\
\hline MCO 1544 & NYDOH 819-85 & & Clinical & 6774373 \\
\hline MCO 1545 & NYDOH $821-85$ & & Clinical & 6776373 \\
\hline MCO 1546 & NYDOH $1306-85$ & & Clinical & 6776373 \\
\hline MCO 1547 & NYDOH $146-86$ & & Clinical (sputum) & 6776373 \\
\hline MCO 1548 & NYDOH $1036-86$ & & Clinical (wound) & 6776373 \\
\hline MCO 1549 & NYDOH $1058-86$ & & Clinical & 6776373 \\
\hline MCO 1551 & NYDOH $277-90$ & & Clinical & 6776373 \\
\hline MCO 1553 & NYDOH 711-92 & & Clinical (eye) & 6776373 \\
\hline MCO 1554 & NYDOH $160-95$ & & Clinical (blood) & 6776373 \\
\hline \multicolumn{5}{|l|}{ Group II strains } \\
\hline MCO 848 & GSU Atl. GD-17 & & $?$ & 6776373 \\
\hline MCO 1439 & ATCC $22995^{\mathrm{T}}$ & Torulopsis kestoni & Air in sugar refinery, United Kingdom & $6776(1,3) 73$ \\
\hline MCO 1441 & ATCC $28873^{\mathrm{T}}$ & Trichosporon appendiculare & Human appendix, Brazil & 6776373 \\
\hline MCO 1464 & ATCC 20296 & C. guilliermondii subsp. galactosa & ? & 6776373 \\
\hline MCO 1550 & NYDOH $1015-88$ & & Clinical (sputum) & 6776373 \\
\hline MCO 1552 & NYDOH 488-92 & & Clinical (vitreous) & 6776373 \\
\hline MCO $1561^{\mathrm{T}}$ & CBS $2022^{\mathrm{TC}}$ & Torula fermentati & Air, Japan & 6776373 \\
\hline \multicolumn{5}{|l|}{ Group III strain } \\
\hline MCO 1440 & ATCC 22949 & & Pigeon & $67(5,7) 6373$ \\
\hline \multicolumn{5}{|l|}{ Group IV strain } \\
\hline MCO 1538 & ATCC 20216 & C. guilliermondii var. soya & $?$ & 6356372 \\
\hline \multicolumn{5}{|l|}{ Group V strain } \\
\hline MCO $1563^{\mathrm{T}}$ & CBS $5256^{\mathrm{T} c}$ & C. guilliermondii var. carpophila & Fig wasp in fruit of Ficus sp. & 6776362 \\
\hline
\end{tabular}

${ }^{a}$ ATCC, American Type Culture Collection, Rockville, Md;; CBS, Centraalbureau voor Schimmelcultures, Delft, The Netherlands; NRRL, Agricultural Research Service Culture Collection, National Center for Agricultural Utilization Research, U.S. Department of Agriculture, Peoria, Ill.; NYDOH, Collection at the New York State Department of Health, Albany; GSU, Donald G. Ahearn, Georgia State University, Atlanta.

${ }^{b}$ Redefined type strain of $Y$. guilliermondii and type strain of $P$. guilliermondii.

c A RAPD pattern was not obtained; the group to which the strain belonged was determined by an isoenzyme pattern analysis.

$\mu \mathrm{g} / \mathrm{gel}$ lane). The enzyme activities analyzed by using $5.0 \%$ (wt/vol) polyacrylamide gels were $\alpha$-mannosidase (EC 3.2.1.24) (350 $\mu \mathrm{g} / \mathrm{gel}$ lane), catalase (EC 1.11.1.6) $(100 \mu \mathrm{g} / \mathrm{gel}$ lane), and superoxide dismutase (EC 1.15.1.1) $(350 \mu \mathrm{g} / \mathrm{gel}$ lane).

RAPD analysis. Yeasts that were cultured as described above for the isoenzyme analysis were centrifuged at $2,000 \times g$ for $10 \mathrm{~min}$, and $200 \mu \mathrm{l}$ of each cell pellet was transferred to a $1.5-\mathrm{ml}$ microcentrifuge tube. The yeasts were washed once with $100 \mathrm{mM}$ Tris hydrochloride ( $\mathrm{pH} 8.0$ ), and then the DNA was extracted and purified. The DNA was prepared for RAPD analysis as described previously (21).

The following primers used for PCR were obtained from J. R. Hobbs, University of British Columbia, Vancouver, British Columbia, Canada: UBC32 (5' GGGGCCTTAA), UBC134 (5'-AACACACGAG), UBC143 (5'-TCGCAGAA CG), UBC147 (5'-GTGCGTCCTC), UBC709 (5'-CCTCCTCCCT), UBC710 (5'-GGTGGTGGGT), UBC711 (5'-CCCTCTCCCT), UBC712 (5'-GGGTGTG
GGT), UBC713 (5' -CCCTCCCTCT), UBC714 (5'-GGGTGGGTGT), UBC715 (5'-CCACCACCCA), UBC716 (5'-GGAGGAGGGA), UBC718 (5'-GGGAG AGGGA), UBC720 (5'-GGGAGGGAGA), UBC721 (5'-CCCTTCCCTC), UBC722 (5'-CCTCTCCCTC), UBC723 (5'-CCCTCTCCTC), UBC725 (5'-GG GTTGGGTG), UBC726 (5'-GGTGTGGGTG), UBC727 (5'-GGGTGTGGT G), UBC728 (5'-GTGGGTGGTG), UBC731 (5'-CCCACACCAC), UBC733 (5'-GGGAAGGGAG), UBC734 (5'-GGAGAGGGAG), UBC736 (5'-GAGGG AGGAG), UBC738 (5'-GGTGGGTGGT), UBC739 (5'-GGAGGGAGAG), UBC740 (5'-GGAGGGAGGA), UBC741 (5'-CCTCCCTCTC), UBC742 (5'-C CTCCCTCCT), UBC743 (5'-CCACCCACAC), UBC744 (5'-CCACCCACCA), UBC745 (5'-GGGAAGAGGG), UBC747 (5'-CCACCAACCC), and UBC754 (5'-GGGTGGTGGT).

When the RAPD analysis was performed, a single 10 -mer primer was used with $C$. guilliermondii DNA in a PCR having a $94^{\circ} \mathrm{C}-36^{\circ} \mathrm{C}-72^{\circ} \mathrm{C}$ cycle; the PCR products were then analyzed by agarose gel electrophoresis (21). 
$\alpha-\operatorname{Man}$

$\begin{array}{llllllllll}1 & 2 & 3 & 4 & 5 & 6 & 7 & 8 & 9 & 10\end{array}$

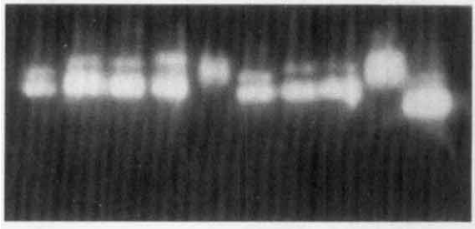

CAT

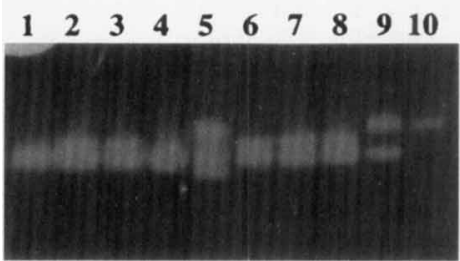

$\alpha$-Glu

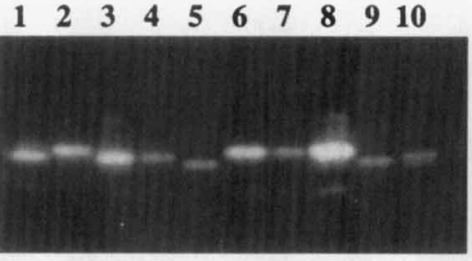

EST

$\begin{array}{llllllllll}1 & 2 & 3 & 4 & 5 & 6 & 7 & 8 & 9 & 10\end{array}$

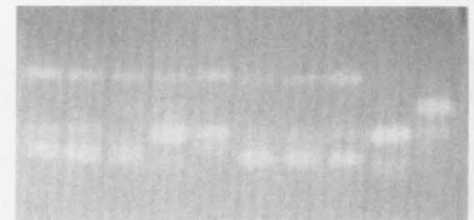

$\beta$-Glu

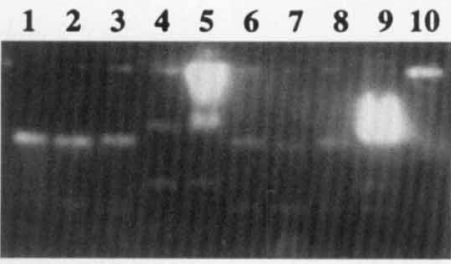

MDH

$\begin{array}{llllllllll}1 & 2 & 3 & 4 & 5 & 6 & 7 & 8 & 9 & 10\end{array}$

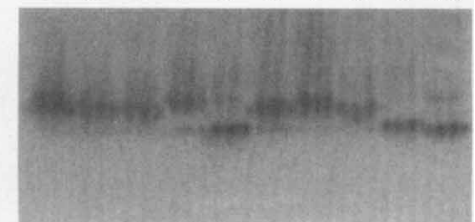

FIG. 1. Isoenzyme patterns produced by members of C. guilliermondii groups I through IV. Lanes 1 through 3 and 6 through 8 , group I strains MCO $1442^{\mathrm{T}}$, MCO 1459, MCO 1536, MCO 1461, MCO 1462, and MCO 1463, respectively; lane 4, group III strain MCO 1440; lanes 5 and 9 , group 2 strains $C$. fermentati MCO 1441 and MCO 1464, respectively; lane 10, group IV strain MCO 1538. $\alpha$-Man, $\alpha$-mannosidase; $\alpha$-Glu, $\alpha$-glucosidase; $\beta$-Glu, $\beta$-glucosidase; CAT, catalase; EST, $\alpha$-esterase; $\mathrm{MDH}$, malate dehydrogenase.

Slime production. Slime production was determined by using a modification of the test established for coagulase-negative staphylococci $(11,12)$ as described by Branchini et al. (6). Briefly, loopfuls of yeast cells from the surfaces of plates containing Sabouraud dextrose agar $(20 \mathrm{~g}$ of D-glucose per liter, $10 \mathrm{~g}$ of neopeptone [Difco] per liter, $15 \mathrm{~g}$ of agar per liter) were inoculated into tubes containing $10 \mathrm{ml}$ of Sabouraud dextrose broth, Sabouraud dextrose broth supplemented with glucose (final concentration, $80 \mathrm{~g} / \mathrm{liter}$ ), YNB (Difco) containing $8 \%$ (wt) vol) glucose, or YPD broth supplemented with glucose (10 g of yeast extract [Difco] per liter, $10 \mathrm{~g}$ of peptone [Difco] per liter, $80 \mathrm{~g}$ of D-glucose per liter). The tubes were incubated at $37^{\circ} \mathrm{C}$ for $24 \mathrm{~h}$ without movement and then in a reciprocal shaker at $\sim 140 \mathrm{rpm}$, after which the broth was removed and the walls of the tubes were stained with safranin. Staining was scored as negative to weak, moderate, or heavy.

Proteinase detection. Secretion of proteinase by $C$. guilliermondii on bovine serum albumin (BSA) agar was tested by using a semiquantitative screening procedure (24) similar to that described by Rüchel et al. (31). The inocula were prepared from 24-h cultures on Sabouraud dextrose agar. Colonies were resuspended in sterile distilled water to a turbidity equivalent to a no. $0.5 \mathrm{McF}$ arland standard. Yeast suspension samples $(5 \mu \mathrm{l})$ were inoculated onto BSA agar plates, and the cultures were incubated at $24^{\circ} \mathrm{C}$ for $3,6,9,12,15$, and 20 days. Proteolysis of BSA was visualized by staining with $0.1 \%$ naphthol blue black (Sigma) in $3.5 \mathrm{M}$ acetic acid and was scored in the manner described by Lin et al. (24). Esterase detection. To detect esterase activity, modified substrate agar plates were used $(32,37)$. The polyoxyethylene sorbitan substrates Tween 20 , Tween 40 , Tween 60, Tween 80, and Tween 85 (Sigma Chemical Co., St. Louis, Mo.) were added separately to Sabouraud liquid modified medium (Difco) to a final concentration of $1 \%(\mathrm{vol} / \mathrm{vol})$. Overnight cultures were transferred to the substrate test medium, and the plates were incubated at $37^{\circ} \mathrm{C}$ in a humid environment and observed at various times for up to 20 days. Lipolytic activity was detected by inspecting the plates for zones of precipitation around the colonies.

Killer activity. The $C$. guilliermondii isolates were tested for killer activity against either Candida albicans MCO 261, an adenine auxotroph (SGY 126: ade2) obtained from D. R. Kirsch, Squibb Institute for Medical Research, Princeton, N.J., or Saccharomyces cerevisiae MCO 182, a uracil auxotroph (TH29A: mnn2 ura3) obtained from C. E. Ballou, University of California, Berkeley. The target yeasts were grown on Sabouraud dextrose agar and then suspended in sterile distilled water, and the turbidity was adjusted to a McFarland standard of $\sim 2$. Then 1-ml portions of the suspensions were spread onto petri dishes containing SD agar $(6.7 \mathrm{~g}$ of YNB [Difco] per liter, $20 \mathrm{~g}$ of glucose per liter, $15 \mathrm{~g}$ of agar per liter), which was buffered at $\mathrm{pH} 4.5$ with $100 \mathrm{mM}$ phosphate-citrate (30), and the excess liquid was removed after $5 \mathrm{~min}$. Each strain of $C$. guilliermondii was streaked onto the dry agar as described previously (30). The plates were incubated at $24^{\circ} \mathrm{C}$ for $48 \mathrm{~h}$ to allow growth of $C$. guilliermondii, and then adenine $(1.2 \mathrm{mg} / \mathrm{ml})$ or uracil $(2.4 \mathrm{mg} / \mathrm{ml}$ ) was added by filling six or seven wells (diameter, $8 \mathrm{~mm}$ ) that had been cut into the agar. Addition of these nutrients enabled the auxotrophic target yeasts to grow. Following an additional 24 to $48 \mathrm{~h}$ of incubation, the plates were inspected for killer activity (30).

Physiological tests. A number of physiological tests were used mainly because their results had been reported to be variable in previous studies on $C$. guilliermondii $(2,26)$. To test for assimilation, yeasts were inoculated into broth containing D-glucose, melibiose, galactitol, glycerol, D-ribose, or D-arabinose as a sole source of carbon. For fermentation studies, D-glucose, D-galactose, methyl$\alpha$-D-glucopyranoside, and raffinose were used, and the cultures were incubated a $30^{\circ} \mathrm{C}(38)$. To test for assimilation of compounds as sole sources of nitrogen, we used an auxanographic method in which a solution of uracil, adenine, potassium nitrate, potassium nitrite, D-glucosamine, L-lysine, thiourea, or creatinine was added to a well cut into yeast carbon base (Difco) agar on which C. guilliermondit had been plated as a lawn (38).

CHROMagar. Isolates were incubated at $30^{\circ} \mathrm{C}$ for 24 to $72 \mathrm{~h}$ on CHROMagar (CHROMagar, Palm Harbor, Fla.). The medium was prepared by mixing CHROMagar powder with distilled water and heating the preparation to boiling which melted the agar; then the medium was poured into petri dishes. The color of the resulting colonies was recorded (29).

Antifungal agent susceptibility test. An antifungal agent susceptibility test was performed by a macrodilution method described by Fromtling et al. (14), with the minor modifications described by Lin et al. (24). The tubes were incubated without shaking at $30^{\circ} \mathrm{C}$ for $48 \mathrm{~h}$. The lowest concentration of an antifungal agent associated with no visible growth in a tube was considered the MIC

Rapid identification kits. The RapID Yeast Plus System (Innovative Diagnostics Systems, Norcross, Ga.) and the MicroScan Rapid Yeast Identification Pane (Baxter Diagnostics, Deerfield, Ill.) were used according to the manufacturers instructions.

\section{RESULTS}

All of the strains produced yeast cells and filaments, usually clearly pseudohyphae, on corn meal agar, although in some cases the filaments were sparse. Initial studies in which isoenzyme analysis was used demonstrated the presence of apparently unrelated organisms among the $C$. guilliermondii strains obtained from the ATCC. While all of the strains were tested, the results obtained for only a selection of the strains and enzymes employed are shown in Fig. 1, in which the ATCC isolates represent four groups. Several strains resembled the type cultures of $C$. guilliermondii and $Y$. guilliermondii, but the patterns obtained for several enzymes, including $\alpha$-mannosidase, $\alpha$-glucosidase, catalase, $\alpha$-esterase, and malate dehydrogenase, placed MCO 1441 and MCO 1464 in a separate group (group II). The strong $\beta$-glucosidase activity visible in Fig. 1 was not observed for all of the isolates in group II. Another distinctive group, group IV, was readily detected by using the isoenzyme patterns obtained for MCO 1538. The API 20C biotype of this strain was quite different from the API $20 \mathrm{C}$ biotypes of the other strains. The single strain in group III, MCO 1440, had features of both group I and group II in some of its enzyme patterns but could not be clearly assigned to one 
CAT

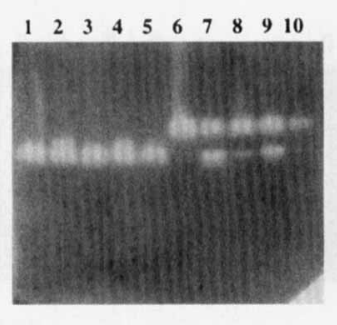

MDH

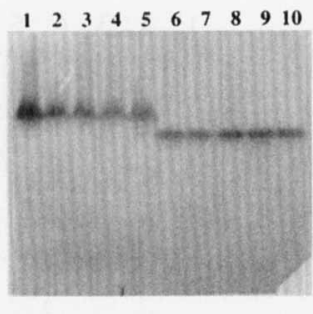

Protein (5\%)

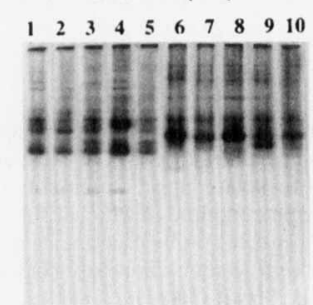

$\alpha-$ Man

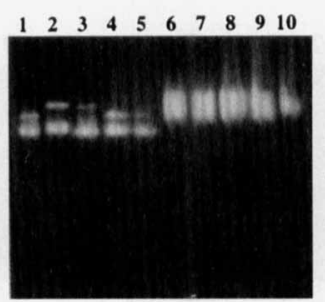

EST

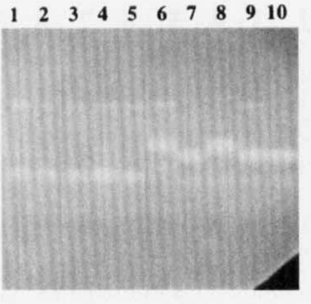

GDH

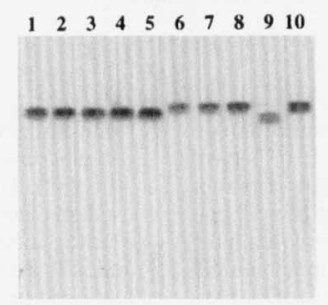

$\alpha$-Glu

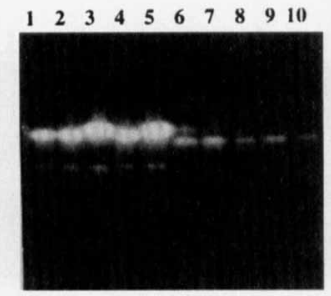

ALP

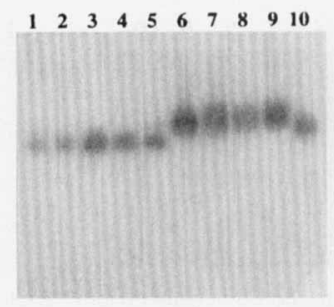

Protein (7.5\%)

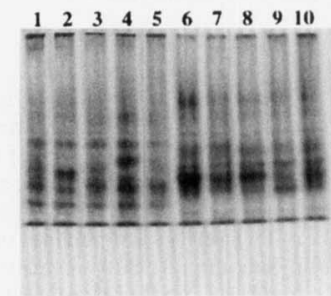

FIG. 2. Isoenzyme patterns produced by members of $C$. guilliermondii groups I and II. Lanes 1 through 5 , group I strains $\mathrm{MCO} 1442^{\mathrm{T}}$, MCO 1525 , MCO 1541 , MCO 1544 , and MCO 1545, respectively; lanes 6 through 10, group II strains MCO 1439, MCO 1464, MCO 1550, MCO 1552, and MCO 848, respectively. CAT, catalase; $\alpha$-Man, $\alpha$-mannosidase; $\alpha$-Glu, $\alpha$-glucosidase; MDH, malate dehydrogenase; EST, $\alpha$-esterase; ALP, alkaline phosphate; GDH, glucose-6-phosphate dehydrogenase.

of these groups. Another isoenzyme-defined group, not shown in Fig. 1, was represented by $C$. guilliermondii var. carpophila MCO $1563^{\mathrm{T}}$ (Table 1).

The clinical isolates, many of which had been sent for identification to the New York State Department of Health, belonged to $C$. guilliermondii groups I and II. Most of these strains had isoenzyme profiles consistent with group I, but the profiles of three of the clinical isolates in the panel, MCO 1441, MCO 1550, and MCO 1552, were group II profiles (Table 1). The distinctive isoenzyme and cellular protein patterns that distinguished group I from group II were produced by both clinical isolates and some non-clinical reference strains (Fig. 2).

The same groups were detected when RAPD profiles were used. The somewhat intermediate nature of group III, which had been noted when the isoenzyme patterns were examined, was confirmed by the RAPD analyses; MCO 1440 (group III) exhibited some similarity to group II when primers UBC710 and UBC739 were utilized, but it did not resemble group II when primer UBC740 was used (Fig. 3). However, when the remaining 31 primers listed in Materials and Methods were used to obtain RAPD patterns for the group III isolate, all but two patterns separated MCO 1440 from strains representing groups I and II (MCO 1459, MCO 1460, MCO 1441, and MCO 1464). The results obtained with primer UBC713 suggested that MCO 1440 has affinity to both group I and group II, and the results obtained with primer UBC715 suggested that MCO 1440 has affinity to group II (data not shown). The group IV strain, MCO 1538, was clearly discriminated from groups I, II, and III in all cases.
A number of different Candida species were compared by RAPD analyses in which primers UBC710, UBC739, and UBC740 (Fig. 4) and primer UBC32 (data not shown) were used. None of these species exhibited a clear resemblance to any $C$. guilliermondii group.

Phenotypic characteristics of groups. A number of tests were performed to distinguish the $C$. guilliermondii groups phenotypically and to investigate characteristics that have been suggested as characteristics that play a role in determining the virulence of pathogenic yeasts. These tests included tests for production of slime and formation of extracellular proteinases.

(i) Slime production. Initially, four media were tested for the ability to detect slime production by $C$. guilliermondii. Slime production was inhibited by shaking the cultures in Sabouraud and YNB broth media that contained $8 \%(\mathrm{wt} / \mathrm{vol})$ glucose. In contrast, slime production was more pronounced in shaken cultures than in unshaken cultures when Sabouraud broth containing 2\% glucose was used. Glucose-enriched YPD broth was quite variable in its support of slime production, both in still cultures and in shaken cultures. Given the well-defined medium provided by unshaken, glucose-enriched YNB broth, this medium was used to screen the strains.

Table 2 shows the slime production results. The majority of the strains in groups I and II produced slime, and slime production was associated with both clinical and environmental isolates. Neither the group III strain nor the group IV strain produced slime.

(ii) Formation of proteinases. Proteinase production was generally weak or not detectable. After 12 days, only MCO 1467 produced a distinct zone, and after 20 days an additional 


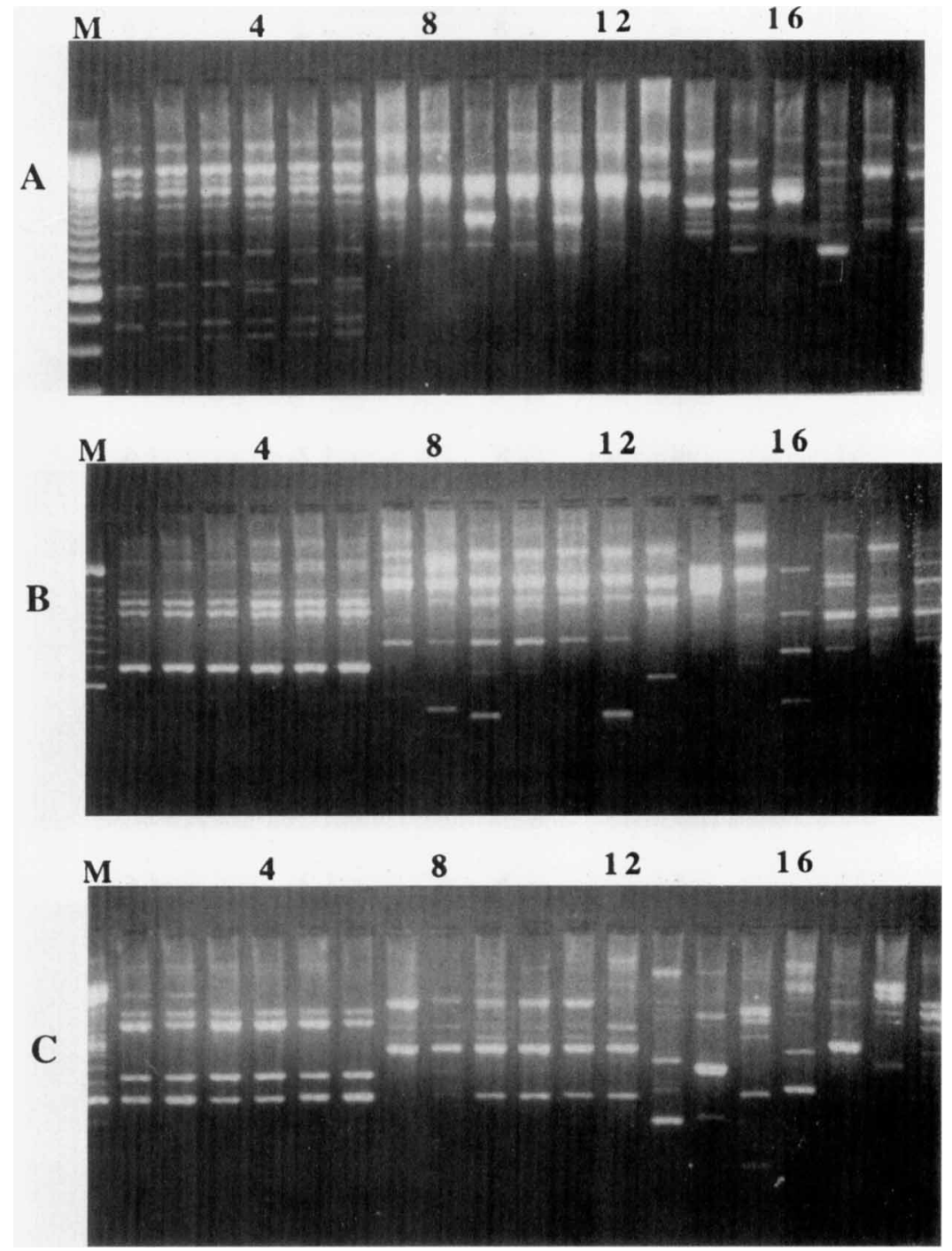

FIG. 3. RAPD patterns obtained for members of $C$. guilliermondii groups and other Candida species by using primers UBC710 (A), UBC739 (B), and UBC740 (C) Lanes 1 through 6, group I strains MCO 1525, MCO 1442 $\mathrm{T}$ MCO 1461, MCO 1541, MCO 1544, and MCO 1545, respectively; lanes 7 through 12 , group II strains MCO 1464, MCO 1439, MCO 1441, MCO 1550, MCO 1552, and MCO 848, respectively; lane 13, group III strain MCO 1440; lane 14, group IV strain MCO 1538; lane 15, Candida famata ATCC 62894; lane 16, Candida lusitaniae ATCC 42720; lane 17, Candida maltosa R-42 (obtained from D. G. Ahearn, Georgia State University); lane 18, C. haemulonii CDC 86-041135 (group I); lane 19, C. haemulonii CDC 90-003593 (group II) (23).

4 of the 23 group I strains appeared to be positive (score, $1+$ ) (24). None of the strains belonging to the other groups were positive after 12 days, although a single group II strain, MCO 1464 , showed evidence of weak activity after 20 days of incubation. In contrast to the limited activity associated with $C$. guilliermondii, after 12 days both Candida parapsilosis MCO 846 and MCO 847 exhibited obvious proteinase activity (score, $2+)(24)$.

Esterase. Minimal esterase activity was detected on agar media containing Tween 20 , Tween 60 , or Tween 80 . Thus, this test did not allow us to distinguish $C$. guilliermondii groups I and II.

Killer activity. Both of the target strains used were auxotrophic mutants. This allowed us to suspend the growth of these organisms for 2 days in an attempt to increase the assay's sensitivity for inhibitory compounds. Weak inhibitory activity was produced by group IV isolate MCO 1538 (Pichia ohmeri) when it was tested against $C$. albicans and $S$. cerevisiae. Weak inhibitory activity was observed with $C$. guilliermondii $\mathrm{MCO}$
1459, MCO 1460, and MCO 1525 (group I strains) when these organisms were tested against $C$. albicans, but no activity was observed when $S$. cerevisiae was the target. The activity was detectable in media buffered at $\mathrm{pH} 4.0,4.5$, and 5.0 but not in media buffered at $\mathrm{pH} 6.0$ or 7.0. These results did not establish a useful phenotype for discriminating $C$. guilliermondii groups I and II.

Physiological tests. In the sugar assimilation and fermentation tests done in broth that were performed to distinguish $C$. guilliermondii group I from group II, the only difference found was a difference in the ability of the strains to ferment Dgalactose after prolonged incubation. After 21 days at $30^{\circ} \mathrm{C}$, all of the group II strains had produced gas, as had $C$. guilliermondii MCO 1538 (group IV) and MCO $1563^{\mathrm{T}}$ (group V), whereas no $C$. guilliermondii group I or III strain had this phenotype. Fermentation of D-glucose was detected within 21 days for all strains belonging to groups I through V.

In tests performed with all of the strains except strains $\mathrm{MCO}$ 1557 through MCO $1563^{\mathrm{T}}$ (see Materials and Methods), 

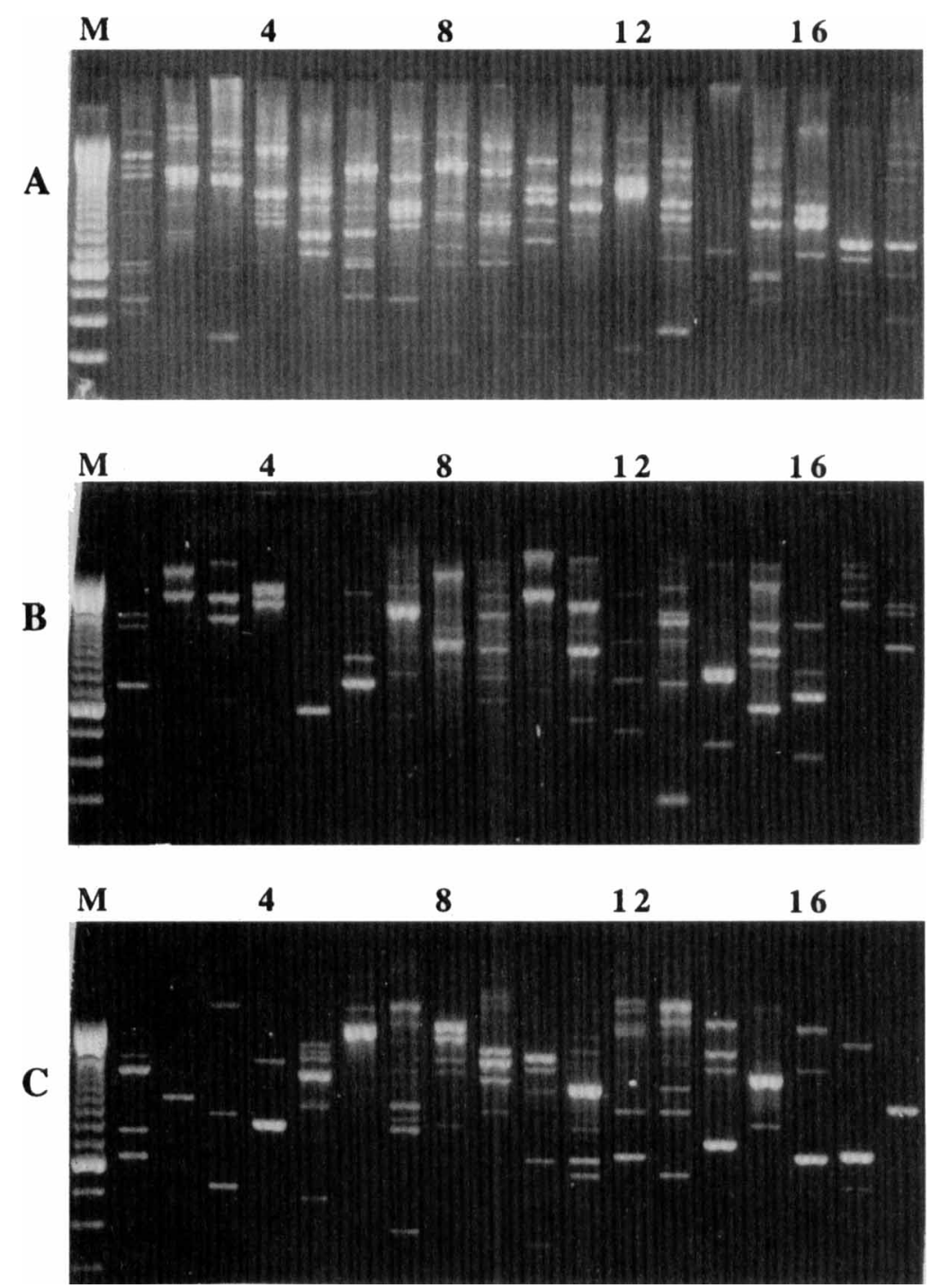

FIG. 4. Comparison of RAPD patterns obtained for different species by using primers UBC710 (A), UBC739 (B), and UBC740 (C). Lane M, 100-bp DNA ladder with the lowest bright band at $600 \mathrm{bp}$; lane $1, C$. guilliermondii group I strain MCO 1525; lane 2, C. guilliermondii group II strain MCO 1439; lane 3, C. guilliermondii group III strain MCO 1440; lane 4, C. guilliermondii group IV strain MCO 1538; lane 5, C. parapsilosis group I strain ATCC 22019; lane 6, C. parapsilosis group II strain MCO 471; lane 7, C. parapsilosis group III strain MCO 429 (24); lane 8, C. haemulonii group I strain CDC 86-041135; lane 9, C. haemulonii group II strain CDC 90-003593; lane 10, Candida famata ATCC 62894; lane 11, Candida intermedia R-666 (obtained from M. G. Rinaldi, University of Texas Health Sciences Center, San Antonio); lane 12, Candida lusitaniae ATCC 42720; lane 13, Candida zeylanoides ATCC 7251; lane 14, Candida ernobii ATCC 60118; lane 15, Candida rhagii ATCC 22983; lane 16, Candida holmii ATCC 22034; lane 17, Candida inconspicua ATCC 16783; lane 18, Candida maltosa R-42.

D-raffinose was fermented by all of the strains except two group I strains (MCO 1463 and MCO 1525), and gas was detected after 6 to 18 days. None of the strains fermented methyl- $\alpha-D-$ glucopyranoside. All of the strains assimilated D-glucose, glycerol, D-arabinose, and dulcitol. The majority of the group I strains ( 20 of the 23 strains tested) and the group III and IV strains were positive for assimilation of glycerol after 3 days, and the remainder of the group I strains were positive after 6 days. In contrast, the group II strains were not positive for glycerol assimilation until after 6 or more days. Assimilation of D-arabinose was typically more rapid with group II strains than with group I strains. Five of the six group II strains were positive after 3 days, and the remaining strain (MCO 1550) was positive after 10 days. Only 2 of the 23 group I strains (MCO 1459 and MCO 1467) were positive for arabinose assimilation after 6 days, and the remainder of the group I strains were positive after 10 to 15 days.
API 20C profiles and characteristics on CHROMagar. Most of the API $20 \mathrm{C}$ profiles (Table 1) appeared to be very similar, and typically these profiles had biocode 6776373 ; the only exception was the profile for $C$. guilliermondii var. soya MCO 1538 (group IV). This strain also appeared to be quite distinct

TABLE 2. Number of strains producing slime

\begin{tabular}{lcccc}
\hline \multirow{2}{*}{ Group } & $\begin{array}{c}\text { No. of strains } \\
\text { tested }\end{array}$ & \multicolumn{3}{c}{$\begin{array}{c}\text { No. of strains with the following } \\
\text { slime production: }\end{array}$} \\
\cline { 3 - 5 } & & Negative to weak & Moderate & Heavy \\
\hline I & 23 & 3 & 16 & 4 \\
II & 6 & 1 & 3 & 2 \\
III & 1 & 1 & & \\
IV & 1 & 1 & & \\
\hline
\end{tabular}


TABLE 3. MICs of amphotericin B, ketoconazole, and 5-fluorocytosine

\begin{tabular}{|c|c|c|c|c|c|c|c|c|c|}
\hline \multirow{2}{*}{$\begin{array}{l}\text { Antifungal } \\
\text { agent }\end{array}$} & \multirow{2}{*}{ Group } & \multirow{2}{*}{$\begin{array}{l}\text { No. of strains } \\
\text { tested }\end{array}$} & \multicolumn{7}{|c|}{ No. of strains with an MIC of: } \\
\hline & & & $0.015 \mu \mathrm{g} / \mathrm{ml}$ & $0.03 \mu \mathrm{g} / \mathrm{ml}$ & $0.06 \mu \mathrm{g} / \mathrm{ml}$ & $0.125 \mu \mathrm{g} / \mathrm{ml}$ & $0.25 \mu \mathrm{g} / \mathrm{ml}$ & $0.50 \mu \mathrm{g} / \mathrm{ml}$ & $2.0 \mu \mathrm{g} / \mathrm{ml}$ \\
\hline \multirow[t]{4}{*}{ Amphotericin B } & I & 23 & & 1 & 4 & 6 & 11 & 1 & \\
\hline & II & 6 & & 1 & 2 & & 1 & 1 & 1 \\
\hline & III & 1 & & & & & & 1 & \\
\hline & IV & 1 & & & & 1 & & & \\
\hline \multirow[t]{4}{*}{ Ketoconazole } & I & 23 & 6 & 12 & 5 & & & & \\
\hline & II & 6 & & 3 & 1 & 2 & & & \\
\hline & III & 1 & & & & & 1 & & \\
\hline & IV & 1 & & 1 & & & & & \\
\hline \multirow[t]{4}{*}{ 5-Fluorocytosine } & I & 23 & 6 & 10 & 7 & & & & \\
\hline & II & 6 & 2 & 3 & 2 & & & & \\
\hline & III & 1 & 1 & & & & & & \\
\hline & IV & 1 & & & 1 & & & & \\
\hline
\end{tabular}

on CHROMagar, on which it produced blue colonies similar to Candida tropicalis colonies. All group I, II, and III strains produced pink colonies.

Antifungal agent susceptibilities. The numbers of strains that were inhibited by different MICs of antifungal agents are shown in Table 3 . The pronounced overlap of MICs that characterized the $C$. guilliermondii group I strains and the group II strains did not allow discrimination of the two yeast groups on the basis of antifungal agent susceptibility.

Rapid identification kits. The results obtained with rapid identification kits are shown in Table 4 . Only 10 group I strains were used in these tests. We found no obvious characteristics that differentiated the biotypes of $C$. guilliermondii belonging to groups I, II, and III. MCO 1538 (group IV; P. ohmeri) had a distinct MicroScan Rapid Yeast Identification Panel biotype with unique scores in the third and fifth positions which reflected a lack of production of esterases hydrolyzing glycyl-Larginine 4-methoxy- $\beta$-naphthylamide and L-histidine $\beta$-naphthylamide, and the group V strain had a unique RapID Yeast Plus score in the third position of the biotype that reflected its failure to produce $\alpha$-glucosidase and its production of an $N$-acetyl- $\beta$-D-galactosaminidase. Otherwise, the biotypes for all strains were similar, and, notably, groups I and II were indistinguishable.

Taxonomy of $\boldsymbol{C}$. guilliermondii group II. The demonstration that there were distinct genetic groups among the strains led us to investigate which of the taxa that had been brought into synonymy with $C$. guilliermondii were found in each of the groups. Table 1 shows that many of the synonyms suggested in The Yeasts: a Taxonomic Study (26) and Yeasts: Characteristics and Identification (2) appeared in the same genetic group as the type strain (C. guilliermondii ATCC 6260). However, Torulopsis kestoni, Torula fermentati, Trichosporon appendiculare, and $C$. guilliermondii subsp. galactosa (a name applied to a strain deposited for patent purposes) represented $C$. guilliermondii group II. Torula fermentati Saito 1922 (33) represents the earliest description of an extant group II culture; the type strains of $E$. negrii Castellani 1912, M. lustigi Castellani et Chalmers 1913, and M. pseudoguilliermondii Castellani et Chalmers 1919 apparently are not extant.

Candida negrii was considered a valid name for $C$. guilliermondii group II, for the original description of $E$. negrii included weak fermentation of galactose as a characteristic (8). We chose not to use this name because we found that in order for fermentation of galactose to be useful for distinguishing $C$. guilliermondii groups I and II, it is necessary to use a temper- ature of $30^{\circ} \mathrm{C}$ for the test. The use of either a higher temperature or a lower temperature does not give clear-cut results (22). It is not clear whether an incubation temperature of $30^{\circ} \mathrm{C}$ was used in the fermentation tests used to describe $E$. negrii (8). Other potential difficulties associated with the use of fermentation tests with D-galactose are discussed below. Given our incomplete understanding of the yeast described as $E$. negrii, a taxon for which no type strain appears to have been deposited, we recommend that yeast isolates which are identified as $C$. guilliermondii and which have RAPD and isoenzyme profiles that place them in group II should be named $C$. fermentati (Saito) Bai (1).

Status of the type strain of $Y$. guilliermondii. Strain CBS 2031 (= MCO $1525=$ ATCC 58070) was defined as the type strain

TABLE 4. Biotypes of $C$. guilliermondii strains as determined with rapid identification kits

\begin{tabular}{|c|c|c|c|}
\hline \multirow{2}{*}{ Strain } & \multirow{2}{*}{ Group } & \multicolumn{2}{|c|}{ Biotype as determined with ${ }^{a}$} \\
\hline & & RYPS kit & MRYIP kit \\
\hline $\mathrm{MCO} 1442^{\mathrm{T}}$ & I & 502307 & 556070654 \\
\hline MCO 1462 & I & 522317 & 717070040 \\
\hline MCO 1463 & I & 506137 & 756072064 \\
\hline MCO $1536^{\mathrm{T}}$ & I & 502237 & 776072044 \\
\hline MCO 1539 & I & 502337 & 774272644 \\
\hline MCO 1540 & I & 502137 & 754270640 \\
\hline MCO 1541 & I & 502337 & 777072640 \\
\hline MCO 1542 & I & 502137 & 774272646 \\
\hline MCO 1543 & I & 502337 & 774270044 \\
\hline MCO 1544 & I & 502337 & 774372646 \\
\hline MCO 848 & II & 506507 & 756072666 \\
\hline MCO 1439 & II & 502337 & 756272646 \\
\hline MCO 1441 & II & 502537 & 755370644 \\
\hline MCO 1464 & II & 506537 & 746070262 \\
\hline MCO 1550 & II & 506117 & 746370646 \\
\hline MCO 1552 & II & 506517 & 756370666 \\
\hline MCO $1561^{\mathrm{T}}$ & II & 502137 & 775372666 \\
\hline MCO 1440 & III & 502307 & 756170640 \\
\hline MCO 1538 & IV & 502107 & 542060640 \\
\hline $\operatorname{MCO} 1563^{\mathrm{T}}$ & V & 505537 & 775370666 \\
\hline
\end{tabular}

${ }^{a}$ RYPS, RapID Yeast Plus System; MRYIP, MicroScan Rapid Yeast Identification Panel. 
of $Y$. guilliermondii (4), and we wish to correct an error in this assignment. Strain CBS 2030 (= MCO $1536=$ ATCC 46036) is the original type strain deposited as Pichia guilliermondii by Wickerham (40), and so this strain should have been considered the type strain of $Y$. guilliermondii.

\section{DISCUSSION}

The use of molecular genetic procedures has resulted in detection of several groups of organisms among the organisms identified as $C$. guilliermondii. Group I is represented by the type strains of $Y$. (= P.) guilliermondii and $C$. guilliermondii. The synonyms for this taxon include $C$. parapsilosis var. tuxtlensis, C. parapsilosis var. tokyoensis, C. guilliermondii var. japonica, Candida mamillae, and Candida amidevorans. This group is common among clinical isolates and has also been detected in the environment (Table 1). No group I strain has been found to ferment galactose within 21 days at $30^{\circ} \mathrm{C}$.

Group II also includes strains that were obtained from clinical specimens. In contrast to the group I strains, the group II strains produced gas during fermentation of galactose; however, this fermentation could be slow and might not become apparent before 21 days of incubation. The type strain of this group is the recently named organism $C$. fermentati CBS 2022 (= MCO 1561), which was originally obtained from the air in Japan (33). Another strain, strain ATCC 22995 (= MCO 1439), which was originally named Torulopsis kestoni, was also obtained from air samples (35). Other synonyms for $C$. fermentati are Trichosporon appendiculare, which was obtained from a clinical source (3), and C. guilliermondii subsp. galactosa (nomen nudum), which was applied to a strain deposited for patent purposes (16). In addition, the original description of $C$. paranensis stated that the species fermented galactose (28); therefore, this name should be considered a synonym of $C$. fermentati.

The distinction between $C$. guilliermondii (group I) and $C$. fermentati (group II) based on delayed fermentation of galactose may not be absolutely reliable. Wickerham reported, in his analysis of 16 sexually reactive strains of $C$. guilliermondii that were presumably group I strains, that galactose was "seldom" fermented (40). Furthermore, in contrast to our findings, other researchers working with group II strains have failed to detect fermentation of galactose despite incubation for 15 or 21 days $(3,33,35)$; however, fermentation of galactose was a significant property that was used in the naming of $C$. guilliermondii subsp. galactosa (16). It is difficult to explain the differences between our results and those of other workers; however, over the years, there may have been slight changes in medium components, or strains may have developed altered phenotypes after being held in culture. Such changes have been recognized commonly; indeed, Castellani stated, "It must be remembered that a certain number of fungi of the genus Monilia have not permanent sugar reactions and can be trained to ferment sugars which they do not ferment when recently isolated, and therefore for purposes of classification and comparison such fungi should be investigated using only recently isolated strains" (10). Similarly, we noted changes in the morphology of the type strain of Candida haemulonii when a laboratory culture was compared to its original description (23).

Groups III through V were studied by using single strains (Table 1). Group III exhibited some similarities in its RAPD and isoenzyme patterns to the group I strain and the group II strain. The taxonomic status of this group requires further investigation. Group IV is represented by a single strain that has been misidentified; the phenotype of this strain is clearly that of Yamadazyma ( $=$ Pichia) ohmeri, and its identity has been confirmed by an isoenzyme analysis performed with a panel of authentic strains which included the type culture of $Y$. ohmeri, NRRL Y-1932 (22).

In view of the reported DNA homology shown by $C$. guilliermondii var. carpophila (group V) and C. guilliermondii group I (27), it is surprising that the isoenzyme patterns of groups I and $V$ are so different (data not shown). Indeed, these patterns suggest that the two groups may represent distinct species, and this could be supported by the rather different $\mathrm{G}+\mathrm{C}$ contents of the group I and V DNAs (27).

Our research shows the value of protocols in which genotypic characteristics are used for classifying yeasts. As we have reported for other Candida species (42), many taxa appear to contain genetically distinct organisms; therefore, descriptions of a taxon's properties can represent combinations of characteristics associated with different species. Genotypic characteristics clarify the number of species present in a taxon and also can be used to validate the application of selected phenotypes to taxonomy under conditions where few phenotypes are available to separate species. In the current example, $C$. fermentati is distinguished from $C$. guilliermondii group I only by the fermentation of galactose, a test that can require up to 3 weeks of incubation before a result is obtained. Although these species are genetically distinct, the results obtained for markers with potential clinical significance, such as production of extracellular proteinase or slime and susceptibility to antifungal agents, showed that they are extremely similar and suggest that they might exhibit similar pathogenic potentials.

Some other clinically important yeast species are discriminated largely by using molecular genetic procedures; thus, Candida dubliniensis resembles $C$. albicans (36). Neither $C$. dubliniensis and $C$. albicans nor $C$. fermentati and $C$. guilliermondii are likely to be routinely discriminated from each other in most clinical laboratories. Because of this, the taxa C. guilliermondii and $C$. albicans may best be considered species complexes rather than discrete species. The development of convenient procedures in which genetic characteristics are used for primary identification of isolates would be of great value for discriminating phenotypically similar organisms that differ genetically.

\section{ACKNOWLEDGMENTS}

This research was supported by a grant from the American Heart Association, Ohio Affiliate.

We thank F.-Y. Bai for providing a copy of his manuscript prior to publication.

\section{REFERENCES}

1. Bai, F.-Y. 1996. Separation of Candida fermentati comb. nov. from Candida guilliermondii by DNA base composition and electrophoretic karyotyping Syst. Appl. Microbiol. 19:178-181.

2. Barnett, J. A., R. W. Payne, and D. Yarrow. 1990. Yeasts: characteristics and identification, 2nd ed. Cambridge University Press, Cambridge, England.

3. Batista, A. C., J. S. Silveira, and G. L. Silveira. 1959. Um novo Trichosporon isolado do apêndice cecal humano. Rev. Assoc. Med. Bras. 5:351-352.

4. Billon-Grand, G. 1989. A new ascosporogenous yeast genus: Yamadazyma gen. nov. Mycotaxon 35:201-204.

5. Bradford, M. M. 1976. A rapid and sensitive method for the quantitation of microgram quantities of protein utilizing the principle of protein-dye binding. Anal. Biochem. 72:248-254.

6. Branchini, M. L., M. A. Pfaller, J. Rhine-Chalberg, T. Frempong, and H. D. Isenberg. 1994. Genotypic variation and slime production among blood and catheter isolates of Candida parapsilosis. J. Clin. Microbiol. 32:452-456.

7. Buesching, W. J., K. Kurek, and G. D. Roberts. 1979. Evaluation of the modified API $20 \mathrm{C}$ system for identification of clinically important yeasts. J. Clin. Microbiol, 9:565-569.

8. Castellani, A. 1912. Observations on the fungi found in tropical bronchomycosis. Lancet i:13-15.

9. Castellani, A. 1912. Note on the importance of hyphomycetes and other fungi in tropical pathology. Br. Med. J. ii:1208-1212. 
10. Castellani, A. 1920. The etiology of thrush. J. Trop. Med. Hyg. 23:17-22.

11. Christensen, G. D., W. A. Simpson, A. L. Bisno, and E. H. Beachy. 1982 Adherence of slime-producing strains of Staphylococcus epidermidis to smooth surfaces. Infect. Immun. 37:318-326.

12. Davenport, D. S., R. M. Massanari, M. A. Pfaller, M. J. Bale, S. A. Streed, and W. J. Hierholzer. 1986. Usefulness of a test for slime production as a marker for clinically significant infections with coagulase-negative staphylococci. J. Infect. Dis. 153:332-339.

13. Diddens, H. A., and J. Lodder. 1942. Die Hefesammlung des "Centraalbureau voor Schimmelcultures." Beiträge zu einer Monographie der Hefearten. II. Die anaskosporogenen Hefen, part 2, p. 246-265. N. V. Noord-Hollandsche Uitgevers Maatschappij, Amsterdam, The Netherlands.

14. Fromtling, R. A., J. N. Galgiani, M. A. Pfaller, A. Espinel-Ingroff, K. F. Bartizal, M. S. Bartlett, B. A. Body, C. Frey, G. Hall, G. D. Roberts, F. B. Nolte, F. C. Odds, M. G. Rinaldi, A. M. Sugar, and K. Villareal. 1993. Multicenter evaluation of a broth macrodilution antifungal susceptibility test for yeast. Antimicrob. Agents Chemother. 37:39-45.

15. Kemker, B. J., P. F. Lehmann, J. W. Lee, and T. J. Walsh. 1991. Distinction of deep versus superficial clinical and nonclinical isolates of Trichosporon beigelii by isoenzymes and restriction fragment length polymorphisms of rDNA generated by polymerase chain reaction. J. Clin. Microbiol. 29:16771683.

16. Kimura, K., K. Takayama, and T. Nakanishi. April 1974. Process for producing citric acid by fermentation. U.S. patent $3,806,414$.

17. Kurtzman, C. P. 1992. DNA relatedness among phenotypically similar species of Pichia. Mycologia 84:72-76.

18. Langeron, M., and P. Guerra. 1938. Nouvelles recherches de zymologie médicale. Ann. Parasitol. Hum. Comp. 16:36-179, 429-476, 481-525.

19. Lehmann, P. F., C.-B. Hsiao, and I. F. Salkin. 1989. Protein and enzyme electrophoresis profiles of selected Candida species. J. Clin. Microbiol. 27: 400-404.

20. Lehmann, P. F., B. J. Kemker, C.-B. Hsiao, and S. Dev. 1989. Isoenzyme biotypes of Candida species. J. Clin. Microbiol. 27:2514-2521.

21. Lehmann, P. F., D. Lin, and B. A. Lasker. 1992. Genotypic identification and characterization of species and strains within the genus Candida by using random amplified polymorphic DNA. J. Clin. Microbiol. 30:3249-3254.

22. Lehmann, P. F., and L.-C. Wu. Unpublished data.

23. Lehmann, P. F., L.-C. Wu, W. R. Pruitt, S. A. Meyer, and D. G. Ahearn. 1993. Unrelatedness of groups of yeasts within the Candida haemulonii complex. J. Clin. Microbiol. 31:1683-1687.

24. Lin, D., L.-C. Wu, M. A. Rinaldi, and P. F. Lehmann. 1995. Three distinct genotypes within Candida parapsilosis from clinical sources. J. Clin. Microbiol. 33:1815-1821.

25. Lodder, J., and N. J. W. Kreger-van Rij. 1952. The yeasts: a taxonomic study, p. 518-524. North-Holland Publishing Co., Amsterdam, The Netherlands,

26. Meyer, S. A., D. G. Ahearn, and D. Yarrow. 1984. Genus 4. Candida Berkhout, p. 585-844. In N. J. W. Kreger-van Rij (ed.), The yeasts: a taxonomic study, 3rd ed. Elsevier Science Publishers B. V., Amsterdam, The Netherlands.

27. Meyer, S. A., and H. J. Phaff. 1972. DNA base composition and DNA-DNA homology studies as tools in yeast systematics, p. 375-386. In A. KockováKratochvílová and E. Minárik (ed.), Yeasts; models in science and technics. Proceedings of the First Specialized International Symposium on Yeasts, Smolenice, June 1-4, 1971. Publishing House of the Slovak Academy of Sciences, Bratislava, Slovakia.

28. Negroni, P., and I. Fischer. 1941. Contribución al conocimiento de la flora micológica (microfitos) del delta de Paraná. Rev. Inst. Bacteriol. Dep. Hig. 10:334-342.

29. Odds, F. C., and R. Bernaerts. 1994. CHROMagar Candida, a new differential isolation medium for presumptive identification of clinically important Candida species. J. Clin. Microbiol. 32:1923-1929.

30. Polonelli, L., C. Archibusacci, M. Sestito, and G. Morace. 1983. Killer system: a simple method for differentiating Candida albicans strains. J. Clin. Microbiol. 17:774-780.

31. Rüchel, R., R. Tegeler, and M. Trost. 1982. A comparison of secretory proteinases from different strains of Candida albicans. Sabouraudia 20:233244

32. Rudek, W. 1978. Esterase activity in Candida species. J. Clin. Microbiol. 6:756-758.

33. Saito, K. 1922. Untersuchungen über die atmosphärischen Pilzkeime. Jpn. J. Bot. 1:1-54.

34. San Millan, R., I. F. Salkin, and P. F. Lehmann. 1996. Genetic heterogeneity detected within Candida guilliermondii, abstr. F-6, p. 74. In Abstracts of the 96th General Meeting of the American Society for Microbiology 1996 American Society for Microbiology, Washington, D.C.

35. Scarr, M. P., and D. Rose. 1966. Study of osmophilic yeasts producing invertase. J. Gen. Microbiol. 45:9-16.

36. Sullivan, D. J., T. J. Westerneng, K. A. Haynes, D. E. Bennett, and D. C. Coleman. 1995. Candida dubliniensis sp. nov.: phenotypic and molecular characterization of a novel species associated with oral candidosis in HIVinfected individuals. Microbiology 141:1507-1521.

37. Tirunarayanan, M. O., and H. Lundbeck. 1968. Investigations on the en zymes and toxins of staphylococci: assay of lipase using Tween as the substrate. Acta Pathol. Microbiol. Scand. 72:263-276.

38. van der Walt, J. P., and D. Yarrow. 1984. Methods for the isolation, main tenance, classification and identification of yeast. IIC. Physiological and biochemical characteristics, p. 76-97. In N. J. W. Kreger-van Rij (ed.), The yeasts: a taxonomic study, 3rd ed. Elsevier Science Publishers B. V., Amsterdam, The Netherlands.

39. van Uden, N., and H. Buckley. 1971. Genus 2. Candida Berkhout, p. 893 1087. In J. Lodder (ed.), The yeasts: a taxonomic study, 2nd ed. NorthHolland Publishing Co., Amsterdam, The Netherlands.

40. Wickerham, L. J. 1966 . Validation of the species Pichia guilliermondii. J. Bacteriol. 92:1269.

41. Wickerham, L. J., and K. A. Burton. 1954. A clarification of the relationship of Candida guilliermondii to other yeasts by a study of their mating types. J. Bacteriol. 68:594-597.

42. Zeng, S., L.-C. Wu, and P. F. Lehmann. 1996. Random amplified polymor phic DNA analysis of culture collection strains of Candida species. J. Med. Vet. Mycol. 34:293-297. 